Neurosurg Focus 18 (1):E4, 2005

\title{
The natural history of intracranial carotid artery atherosclerosis
}

\author{
Ricardo J. Komotar, M.D., J Mocco, M.D., DaVid A. Wilson, B.S., \\ E. Sander Connolly JR., M.D., Sean D. Lavine, M.D., and Philit M. Meyers, M.D. \\ Department of Neurological Surgery, Columbia University, New York, New York
}

\begin{abstract}
Intracranial atherosclerosis is the cause of a significant number of strokes. Despite maximal medical therapy, this disease continues to carry a poor prognosis. The authors reviewed studies in which the outcomes after conservative management in patients with intracranial carotid artery atherosclerosis were reported. Analysis of the literature demonstrates that maximal medical therapy frequently fails with this disease, leaving patients at high risk for cerebral infarction and death. A better understanding of the pathophysiological aspects and natural history of this condition may serve to guide clinical decision making and the choice of therapeutic options in this patient population.
\end{abstract}

\section{KEY WORDS - carotid artery - intracranial atherosclerosis - natural history • stenosis}

Cerebrovascular disease is the most common lifethreatening neurological event in the US. ${ }^{2}$ Intracranial atherosclerosis is responsible for approximately 40,000 of these events per year, representing $10 \%$ of all ischemic strokes. ${ }^{11}$ In general, intracranial atherosclerosis is associated with several risk factors, many of which are the same as those for coronary artery disease. These include noncaucasian race, diabetes, hypertension, hypercholesterolemia, and cigarette smoking. ${ }^{11,12,15}$ Unfortunately, there are currently few treatment options for intracranial atherosclerosis, and despite maximal medical therapy, the prognosis for patients with this condition remains poor.

Endovascular procedures, which have recently gained popularity in the treatment of intracranial atherosclerosis, have tremendous potential to alter the current standard of care for cerebrovascular disease. These techniques, however, carry substantial risks, and their benefit must be weighed against the prognosis for conservative management in this patient population. In this paper we critically review relevant studies of medically managed intracranial CA atherosclerosis in an attempt to understand better the pathophysiological features and natural history of this condition.

\section{Pathophysiological Features of Intracranial Atherosclerosis}

The CA is the most frequent site of intracranial stenosis, accounting for approximately $50 \%$ of cases. ${ }^{1}$ The overwhelming majority of these are diagnosed when the patient presents with an acute ischemic event, either

Abbreviations used in this paper: $\mathrm{CA}=$ carotid artery; $\mathrm{ICA}=$ internal CA; TIA $=$ transient ischemic attack. stroke or a TIA. Published data in studies in which transcranial Doppler ultrasonography and/or cerebral angiography were used have shown intracranial stenoses to be dynamic lesions that may undergo progression, regression, or remain stable during the follow-up period with medical treatment alone. The factors associated with atherosclerotic progression, however, have yet to be elucidated. In addition, current imaging modalities do not accurately predict the natural history of these lesions, leaving clinicians with difficult management decisions.

Our understanding of the pathological features of atherosclerotic plaque is based largely on autopsy studies. From these investigations, it appears that plaque morphology may be important in the setting of acute cerebrovascular syndromes. Two types of plaques exist; these are white and yellow. White plaques may be viewed as stable lesions with thick fibrous caps and low distensibility. In contrast, yellow plaques may be viewed as unstable, with thin fibrous caps, a lipid-rich core, and high distensibility. In comparison with white plaques, yellow plaques are more commonly found in the setting of acute coronary syndromes, indicating that yellow plaques have a higher level of mechanical vulnerability. Yellow plaques, however, become white plaques over time, potentially representing a healing process. Unfortunately, in vivo morphological data are still lacking because of limitations in angioscopic and ultrasonic technology.

Intracranial atherosclerosis may lead to stroke by three mechanisms: 1) perfusion failure; 2) local thrombosis with arterioarterial thromboembolism; and 3) occlusion of penetrating arteries. Each of these mechanisms results in different pathophysiological features with relevance to endovascular treatment. Perfusion failure occurs when the 
collateral circulation is unable to compensate for a severe stenosis, leading to a reduction in distal blood flow. In this scenario, autoregulation allows reflex vasodilation to occur, thereby maintaining cerebral blood flow. Furthermore, the brain parenchyma is able to increase oxygen extraction in an attempt to maintain normal cerebral metabolism. Once these two compensatory pathways reach their limit, however, further decrement in perfusion leads to stroke. Patients with perfusion failure due to intracranial atherosclerosis may represent a subgroup of individuals for whom endovascular therapy has the greatest chance of being effective.

The second mechanism by which intracranial atherosclerosis may cause stroke is local thrombus formation associated with a preexisting atherosclerotic plaque. In this scenario, thrombosis may not only substantially occlude the parent vessel, but it may also result in arterioarterial embolization. These patients' disease can be effectively managed with antithrombotic treatment, statin medications, and angiotensin-converting enzyme antagonists to prevent local thrombosis and promote plaque stabilization.

The third mechanism by which intracranial atherosclerosis may cause stroke is occlusion of the origin of small arteries. Perforating arteries at risk include the lenticulostriate vessels off the middle cerebral arteries, thalamogeniculate vessels off the posterior cerebral arteries, and the median perforating and circumferential arteries off the basilar artery. Occlusion of these small vessels results in stroke in the deep structures within the cerebral hemispheres or brainstem. Therefore, atherosclerotic stenosis of large intracranial arteries may be associated with infarction of cerebral territories supplied by their perforating vessels. Such patients need to be thoroughly examined because endovascular therapy involving angioplasty may completely obstruct bordering arteries and lead to adverse outcomes.

\section{Medical Management}

Intracranial atherosclerosis is medically managed in a fashion analogous to that of systemic atherosclerosis, with the control of risk factors, use of antithrombotic medications, statins, and angiotensin-converting enzyme inhibitors. Endovascular therapy is considered only after maximal medical regimens have failed and patients have suffered recurrent TIA or strokes. Maximal medical regimens involve therapeutic doses of aspirin, ticlopidine, clopidogrel, warfarin, or intravenous heparin., ${ }^{713}$ Patients who continue to be symptomatic while receiving antiplatelet medications are frequently switched to anticoagulant drugs. The efficacy of aspirin compared with warfarin, however, is still controversial, with previous investigations demonstrating conflicting results. ${ }^{7,10,13}$

Recently, a prospective randomized trial that sought to clarify this controversy, the Warfarin-Aspirin Symptomatic Intracranial Diseases Trial, was prematurely halted by the Safety Monitoring Committee after warfarin was associated with a higher incidence of hemorrhage. At the time of the trial's termination, 569 patients had been studied with a mean follow-up duration of 1.7 years, and no difference in efficacy was detected between the two treatments. ${ }^{5,6}$ Currently, another randomized trial specifically addressing the issue of proper medical management of symptomatic intracranial atherosclerosis, the Aspirin Versus Anticoagulants in Symptomatic Intracranial Stenosis trial, is underway. We hope that the results of this study will clarify this topic and delineate the most efficacious management of the disease in this patient population.

\section{Prognosis of Patients With Intracranial CA Atherosclerosis}

In the largest retrospective series, Borozan, et al., ${ }^{4}$ reviewed the outcomes in 93 patients with angiographically documented intracranial stenosis of at least $20 \%$. Seventyone patients had unilateral and 22 had bilateral disease; $24 \%$ were symptomatic. Over a mean follow-up period of 22.5 months, $23 \%$ of patients died; cardiac disease was the cause of death in six individuals (6.5\%). The annual ipsilateral stroke and mortality rates were 5.1 and $10.3 \%$, respectively. These individuals were identified among 885 consecutive patients in whom cerebral angiograms were obtained.

Marzewksi, et al., ${ }^{9}$ published the first detailed review regarding the outcomes in 66 patients with angiographically documented intracranial ICA stenosis of at least $50 \% ; 41 \%$ of these patients were symptomatic. Over a mean follow-up duration of 3.9 years, $12.1 \%$ of patients experienced an isolated TIA in the ICA territory and $15 \%$ suffered a stroke. In this study, $50 \%$ of patients died during the follow-up period; cardiac disease was the cause of death in 18 individuals (27.2\%). The ipsilateral stroke rate was $3.1 \%$ annually.

Another retrospective series was published by Craig, et al. ${ }^{8}$ These authors reviewed the outcomes in patients with angiographically documented intracranial stenosis of at least $30 \%$. Of these 58 patients, $81 \%$ were symptomatic. After a mean follow-up duration of 30 months, the authors found that $43 \%$ of patients experienced an isolated TIA or stroke in the ICA territory. In addition, $43 \%$ of patients died during the follow-up period; cardiac disease was the cause of death in 11 individuals (19\%). The annual ipsilateral stroke and mortality rates were 7.6 and $17.2 \%$, respectively. Of interest, there were no differences between the death rates in symptomatic (42\%) and asymptomatic $(45 \%)$ patients.

Bogousslavsky ${ }^{3}$ reviewed the outcomes in 22 patients with angiographically documented intracranial stenosis of at least $30 \%$. Over a mean follow-up period of 40.4 months, $32 \%$ of patients died; cardiac disease was the cause of death in six individuals $(27.3 \%)$. The annual ipsilateral stroke and mortality rates were 8.1 and $9.5 \%$, respectively.

Wechsler, et al., ${ }^{14}$ reviewed the outcomes in 15 patients with angiographically documented intracranial stenosis of at least $50 \% ; 80 \%$ of these patients were symptomatic. Over a mean follow-up period of 51 months, $20 \%$ of patients suffered a stroke in the ICA territory. In this study, $20 \%$ of patients died during the follow-up period; cardiac disease was the cause of death in two individuals (13.3\%). The annual ipsilateral stroke and mortality rates were 3.1 and $4.7 \%$, respectively. An important finding in this study was that the initial clinical presentation correlated with the presence of hemodynamically significant stenosis; $71 \%$ of symptomatic patients had evidence of impaired flow on 
angiography. In comparison, only $20 \%$ of asymptomatic patients had similar findings. The authors hypothesized that the strokes were the result of embolism distal to the stenosis and that TIAs were the result of slow flow.

The aforementioned studies reveal annual mortality and ipsilateral stroke rates ranging from 4.7 to $17.2 \%$ and 3.1 to $7.6 \%$, respectively. Nevertheless, these investigations have limitations that must be taken into account when interpreting their results. First, each is subject to retrospective bias due to the study design. Second, the patient populations are highly selective and variable, with availability of cerebral angiography studies as an entry criterion and degrees of stenosis ranging from 20 to $70 \%$. Third, the cohorts are small and heterogeneous, and the severity of ipsilateral stroke is not clearly defined. Regardless of their limitations, however, these studies indicate that patients with intracranial CA atherosclerosis are at high risk for recurrent stroke or death and that medical management frequently fails in their treatment. Future prospective investigations are needed to clarify the prognosis for these patients.

\section{CONCLUSIONS}

Despite maximal medical management, intracranial CA atherosclerosis continues to portend a poor prognosis. Conservative management frequently fails in this disease, leaving patients at high risk for cerebral infarction and death. Given the natural history of this condition, advancements in its treatment, particularly those related to endovascular methods, are of the utmost importance.

\section{References}

1. Akins PT, Pilgram TK, Cross DT III, et al: Natural history of stenosis from intracranial atherosclerosis by serial angiography. Stroke 29:433-438, 1998

2. Barnett HJM (ed): Stroke. Pathophysiology, Diagnosis, and Management, ed 3. New York: Churchill Livingston, 1998
3. Bogousslavsky J: Prognosis of carotid siphon stenosis. Stroke 18:537, 1987

4. Borozan PG, Schuler JJ, LaRosa MP, et al: The natural history of isolated carotid siphon stenosis. J Vasc Surg 1:744-749, 1984

5. Caplan LR: Advances in stroke research: basic science, treatment, and clinical trials outcomes. Rev Neurol Dis 1:91-94, 2004

6. Chimowitz M, Lynn M, Howlett-Smith H, et al: WarfarinAspirin Symptomatic Intracranial Disease (WASID) Trial: final results. Stroke 35:235, 2004 (Abstract)

7. Chimowitz MI, Kokkinos J, Strong J, et al: The Warfarin-Aspirin Symptomatic Intracranial Disease Study. Neurology 45: 1488-1493, 1995

8. Craig DR, Meguro $\mathrm{K}$, Watridge $\mathrm{C}$, et al: Intracranial internal carotid artery stenosis. Stroke 13:825-828, 1982

9. Marzewski DJ, Furlan AJ, St. Louis P, et al: Intracranial internal carotid artery stenosis: longterm prognosis. Stroke 13: 821-824, 1982

10. Mohr JP, Thompson JL, Lazar RM, et al: A comparison of warfarin and aspirin for the prevention of recurrent ischemic stroke. N Engl J Med 345:1444-1451, 2001

11. Sacco RL, Kargman DE, Gu Q, et al: Race-ethnicity and determinants of intracranial atherosclerotic cerebral infarction. The Northern Manhattan Stroke Study. Stroke 26:14-20, 1995

12. Segura T, Serena J, Castellanos M, et al: Embolism in acute middle cerebral artery stenosis. Neurology 56:497-501, 2001

13. Thijs VN, Albers GW: Symptomatic intracranial atherosclerosis: outcome of patients who fail antithrombotic therapy. Neurology 55:490-497, 2000

14. Wechsler LR, Kistler JP, Davis KR, et al: The prognosis of carotid siphon stenosis. Stroke 17:714-718, 1986

15. Wityk RJ, Lehman D, Klag M, et al: Race and sex differences in the distribution of cerebral atherosclerosis. Stroke 27: 1974-1980, 1996

Manuscript received November 19, 2004.

Accepted in final form December 6, 2004.

Address reprint requests to: Philip M. Meyers, M.D., Columbia University College of Physicians and Surgeons, Neurological Institute of New York, 710 West 168th Street, Room 423, New York, NY 10032. email: pmm2002@columbia.edu. 